\title{
Fatigue Mediated Lattice Rotation in Al Alloys
}

\author{
R. Goswami ${ }^{1}$, S. B. Qadri and C.S. Pande \\ Division of Materials Science and Technology, Naval Research Laboratory, Washington \\ DC 20375
}

\begin{abstract}
A significant lattice rotation in the plastic zone of a fatigue crack at room temperature in highly oriented Al 1100 and Al 7075 alloys has been observed by employing X-ray diffraction (XRD) and transmission electron microscopy (TEM). The intensity of 111 peak gradually increases as compared to the intensity of 200 peak as we approach the crack, suggesting lattice rotation. The texture was seen to change gradually from the cubic, away from the crack, to a more random orientation close to the crack. We ascribe the lattice rotation to the increase in dislocation density in the plastic zone. The estimated average angle of rotation is approximately $11^{\circ}$, which is sufficient to disturb the cube texture close to the crack. In addition, the observed plastic zone, as estimated by the lattice rotation, is order of magnitude higher as compared to the theoretically predicted plastic zone. This is of great importance, as it leads to a new insight of the underlying physics of the deformation process in the plastic zone under cyclic loading.
\end{abstract}

Key words: Lattice Rotation, Microstructure, Dislocations networks, Twist boundaries, TEM, Fatigue Crack growth

${ }^{1}$ Corresponding Author: Ramasis Goswami, e-mail: ramasis.goswami@nrl.navy.mil

(C) 2017. This manuscript version is made available under the Elsevier user license http://www.elsevier.com/open-access/userlicense/1.0/ 


\section{Introduction}

Although fatigue damage of metals is one of the most important modes of mechanical failure of structures, its underlying mechanism is still not been fully understood. This paper provides a new insight into an aspect of fatigue that is often not investigated. We refer here to a significant lattice rotation across a number of grains close to the crack that accompanies the fatigue process at room temperature. The magnitude and nature of this rotation under fatigue loading till now was unknown. On the other hand, in the monotonic tension test, it is known that grains rotate with increasing applied load due to plastic deformation. In addition, grain rotation has been observed experimentally for polycrystalline materials [1-6] during annealing and recrystallization, and creep deformation at high homologous temperatures. Several theories, such as dislocation glide of a large number of dislocation across several grains [1], grain boundary sliding and grain boundary diffusion have been proposed to explain the mechanisms of grain rotation $[1,2]$ in these cases. For nanocrystalline grains, grain rotation has been reported in different systems at relatively low temperatures [7-14]. A recent experimental observation in nanocrystalline Pt suggests that the rotation is mediated by climb of grain boundary dislocations. The change in misorientation angle between grains has been shown to be associated with the Frank-Bilby dislocation content in the grain boundary [9]. In addition, it is known that the plastic deformation under applied monotonic loading

leads to texture formation in metals, which is associated with shape change of individual grain and the rotation of crystallographic grain orientation. 
It is realized that the lattice rotation during deformation may be related to the redistribution of dislocations and slip processes. In case of fatigue loading, the distribution of dislocations in low and high stacking fault energy materials is different. In relatively low-stacking fault energy materials, such as $\mathrm{Cu}$ and stainless steel $[15,16]$, the redistribution of dislocations leads to cell structures made of dislocation dipoles in the plastic zone. Recently, an attempt has been made to evaluate the dislocation configurations from the rotation angle spread of 1 to $2^{\circ}$ within a grain under monotonic and fatigue loading [17] using diffraction contrast tomography (DCT) for commercially pure $\mathrm{Fe}$, and also to map sub-grain rotation in the deformation region using electron back-scattered diffraction (EBSD) imaging in 304 stainless steel around the corner of a Vickers indentation [18]. In addition, X-ray Laue diffraction scanning has been used to study geometrical necessary dislocations in $\mathrm{Cu}$ during accumulation of fatigue cycles [19].

In high-stacking fault energy material, such as $\mathrm{Al}$, the rearrangement and accumulation of dislocation are entirely different and could lead to different behavior in the plastic zone. The present work deals with the lattice rotation across several grains observed in the plastic zone during fatigue crack growth at room temperature in Al 1100 (H14) and Al 7075 (T7). These observations were made by employing a series of X-ray diffraction (XRD) at different locations from the crack. Transmission electron microscopy (TEM) was then used to characterize the plastic zone and dislocations. We show that the significant lattice rotation under fatigue loading leads to a non-localized deformation behavior, which will provide new insight as to the energy dissipation mechanisms in the plastic zone. In addition, the lattice rotation may also alter the mode of crack propagation, 
which has implication for the modeling of the fatigue life prediction.

\section{Experimental}

Fatigue crack growth tests were performed in vacuum $\left(<6 \times 10^{-6} \mathrm{~Pa}\right)$ background pressure at a cyclic load frequency of $10 \mathrm{~Hz}$ using a sine waveform and a load ratio of 0.10 on compact tension (CT) specimens made of cold rolled-Al 1100 (H14) and Al 7075-T7 alloys. Fig. 1 (a) shows the typical growth rate per cycle as a function of the difference in stress intensity factor, $\Delta \mathrm{K}$, for $\mathrm{Al} 1100(\mathrm{H} 14)$. The part of the $\mathrm{CT}$ specimen containing a fatigue crack is shown in Fig.1 (b). X-ray diffraction measurements were taken in a spot mode along the three dotted lines as shown in the CT specimen. For Al 1100 (H14) and Al 7075-T731, grains were observed to be elongated or pancake shaped parallel to the rolling direction and are approximately $100 \mu \mathrm{m}$ long and $25 \mu \mathrm{m}$ wide (see Fig 1c). In order to study the characteristics of plastic zone and the dislocation configurations, the crack growth was arrested at a different crack lengths corresponding to a certain $\Delta \mathrm{K}$ close to the threshold as well as in the Paris regime. $\mathrm{X}$-ray diffraction was then obtained using a Rigaku $18 \mathrm{~kW}$ generator and a high resolution powder diffractometer perpendicular to the crack length at number of locations along the three dotted lines in the CT specimen. The locations are close to the crack, and 5, 10, 15, 20 and $25 \mathrm{~mm}$ from the crack on both sides of the crack. The spot size of $1 \mathrm{~mm}$ wide window was used to collect the data at different locations. For X-ray mapping, an area of

$6 \times 3 \mathrm{~cm}^{2}$ was scanned around the crack with a spot size of $1 \mathrm{~mm}$ and a separation of $1 \mathrm{~mm}$ between two spots. For TEM observations, $3 \mathrm{~mm}$ diameter cylinders close to crack were cored out. TEM samples were prepared by initially polishing the arc-cut disk samples 
mechanically and finally by thinning in an ion mill with a gun voltage of $4 \mathrm{kV}$ and a sputtering angle of $10^{\circ}$. Tecnai $\mathrm{G}^{2}$ analytical transmission electron microscopes operating at $300 \mathrm{keV}$ was used to characterize the microstructure and dislocations.

\section{Results}

A series of x-ray diffraction (XRD) patterns were obtained from Al 7075 (T7) at different locations, close to the crack and 5, 10, 15, 20 and $25 \mathrm{~mm}$ from the crack, showing the relative variations of 111 and $200 \mathrm{Al}$ peaks (Fig. 2). We observe that the intensity of 111 and 200 peaks are approximately equal $25 \mathrm{~mm}$ away from the crack, suggesting that more grains are 200 oriented. The cold rolled Al alloys usually show cube texture, and in the present case, the 200 peak intensity is relatively stronger as compared to the 111 intensity away from the crack. The relative intensity variation of 111 and 200 peaks, however, gradually changes as we approach the crack. In fact, the intensity of 111 peak gradually increases as compared to that of the 200 peak. We observe similar trend on the intensity variation for $\mathrm{Al} 1100(\mathrm{H} 14)$, although the cube texture in $\mathrm{Al} 1100(\mathrm{H} 14)$ is stronger than that of $\mathrm{Al} 7075$ (T7).

As the intensity of 111 and 200 peaks gradually changes from the crack, one would be able to construct a map around the crack to get the length of the plastic zone. Fig. 3 is a XRD map, obtained from the intensity of 111 peak around a crack in Al 1100 (H14), showing the intensity variation is significantly higher close to the crack. For greater comparison, the integrated intensity ratio of 111 to 200 was presented in Fig. 4 as a function of position, close to the crack and 5, 10, 15, 20 and $25 \mathrm{~mm}$ at either side of the 
crack, for both alloys. For Al 1100 (H14), the peak ratio of 111 to 200 increases from 0.26 at $15 \mathrm{~mm}$ from the crack to 0.585 close to the crack, implying that the change of peak ratio is approximately $125 \%$. On the other hand, for $\mathrm{Al} 7075 \mathrm{~T}(7)$, the peak ratio increases by $175 \%$. This is the main result and it clearly shows that, as we approach the crack, more grains are oriented suitably for diffraction from $\{111\}$ planes as compared to $\{200\}$ planes. This indicates that considerable lattice rotation across several grains takes place close to the crack as a result of fatigue crack growth at room temperature.

We estimate here quantitatively how the preferred orientation changes as a function of distance from the crack using March-Dollase approach [20,21]. The orientation can be estimated quantitatively using the March-Dollase parameter, $r$, which can be written as [20,21]:

$$
r=\left[\frac{\sin ^{2} \alpha}{\left(\left(k / k_{p}\right)^{2 / 3}-\cos ^{2} \alpha\right)}\right]^{1 / 3}
$$

where $\alpha$ is the angle between the plane of preferred orientation and a comparison plane, $\mathrm{k}$ is the observed intensity ratio of the diffraction peaks of these planes, and $\mathrm{k}_{\mathrm{p}}$ is the corresponding intensity ratio of the two planes from the random powder spectrum. The parameter, $r$, is related to the fraction of crystallites showing a preferred orientation along a plane. A complete preferred orientation corresponds to $\mathrm{r}=0$, while a fully random orientation will give $r=1$. For Al $7075 \mathrm{~T}(7)$, the parameter, $r$, turns out to be $0.75,0.84$, $0.92,0.95$ and 0.999 at a distance of $25,15,10,5$ and $0.0 \mathrm{~mm}$ from the crack, respectively, indicating that it gets more random close to the crack. Similarly for Al 1100 
(H14), the $\mathrm{r}$ parameter varies from $0.54,0.553,0.569$ and 0.67 at a distance of $15,10,5$ and $0.0 \mathrm{~mm}$ from the crack, respectively.

The lattice rotation is a mode of plastic deformation and it can be used to estimate the size of the plastic zone by noting the change in intensity ratio. For Al 1100, the intensity ratio (111/200) increases considerably within $5 \mathrm{~mm}$ at either side of the crack. Thus, the plastic zone size (radius) is around $5 \mathrm{~mm}$ (see Fig. 4). However, the plastic zone size for $\mathrm{Al} 7075$ (T7) is $15 \mathrm{~mm}$, considerably higher as compared to $\mathrm{Al} 1100$. The experimentally measured plastic zone can be compared with the calculated plastic zone size using the equation given below. Here we use plane stress condition to estimate the upper limit of the zone. Usually, the fatigue crack growth produces two plastic regions due to forward and reverse loadings. The forward plastic zone $\left(\mathrm{r}_{\mathrm{f}}\right)$ is estimated by the following expression [22]:

$$
\mathrm{r}_{\mathrm{f}}=(1 / 2 \pi)^{*}\left(\mathrm{~K}_{\max } / \sigma_{\mathrm{ys}}\right)^{2}
$$

where $\sigma_{y s}$ is the yield strength, $K_{\max }$ is the maximum stress intensity factor. As the lattice rotation is a mode of plastic deformation, the distance at either side of the crack through which the rotation is observed has been estimated as plastic zone. The zone radius calculated using Eq. 2 with R-ratio $=0.1, \sigma_{\mathrm{ys}}=105 \mathrm{MPa}$ and $\Delta \mathrm{K}_{\max }=12 \mathrm{MPa} \mathrm{m}^{1 / 2}$ turns out to be $3 \mathrm{~mm}$. On the other hand, the calculated zone radius for Al 7075 (T7) for Rratio $=0.1, \sigma_{\mathrm{ys}} \approx 350 \mathrm{MPa}$ and $\Delta \mathrm{K}_{\max }=13 \mathrm{MPa} \mathrm{m}{ }^{1 / 2}$ is $0.3 \mathrm{~mm}$. The experimentally estimated plastic zone (radius) is $15 \mathrm{~mm}$ for $\mathrm{Al} 7075$ (see Fig. 4), which is an order of 
magnitude higher than the calculated plastic zone using Eq. 2. Note the plastic zone size (forward plastic zone in plain stress condition) estimated by eq. 2 is the upper limit. All these suggest that the present theory severely underestimates the plastic zone size.

The large plastic zone observed in Al 7075 as compared to Al 1100 could be associated with the higher dislocation glide distance across several grains. Note that the average width of the pancake sized grain is $25 \mu \mathrm{m}$ and the observed plastic zone radius is $15 \mathrm{~mm}$. To understand this behavior, we studied the dislocation density and the dislocation configuration as function of distance from the crack. The dislocation density was analyzed using the full-width at half maxima (FWHM) of the 111 peak. Dislocations, strain, bending and crystal size contribute to the measured width of a peak. If it is assumed that a curve is Gaussian in shape and that the intensities of various components are Gaussian in distribution, then the FWHM of the measured curve, $\beta_{\mathrm{M}}$, is given by [2325]:

$$
\beta_{\mathrm{M}}^{2} \approx \beta_{\mathrm{S}}^{2}+\beta_{\mathrm{B}}^{2}+\beta_{\mathrm{D}}^{2}+\beta_{\mathrm{St}}^{2} \ldots \ldots \text { (3) }
$$

where $\beta s, \beta_{B}, \beta_{D}$ and $\beta_{S t}$ are FDHMs due to size, bending, dislocations and strain, respectively. We are interested in estimating the change in dislocation density in the plastic zone as compared to density away from the zone. We observe that the contribution from strain is small as the lattice parameter (a), calculated using 111 peak, does not appreciably change $(\Delta \mathrm{a} / \mathrm{a}=0.0015)$ around the crack tip. Assuming that the dominant contribution to the width of 111 peak is due to dislocations, as the average grain size (width $=25 \mu \mathrm{m}$ and length $=100 \mu \mathrm{m}$ ) is relatively large and the bending is small, the 
density of dislocations, $\rho$, can then be calculated from the FWHM by using the following expression $[23,25]$ :

$$
\rho=\beta_{\mathrm{D}}^{2} / 4.35 \mathrm{~b}^{2}
$$

where $\mathrm{b}$ is the Burger's vector. The apparent dislocation density obtained from Eq. 4 using the peak width of 111 peak as a function of distance from crack is given in Fig. 5 . The density of dislocation obtained from the FWHM of 111 peak away $(25 \mathrm{~mm})$ from the crack is $\approx 2.35 \mathrm{x} 10^{13} \mathrm{~m}^{-2}$ to $2.6 \mathrm{x} 10^{13} \mathrm{~m}^{-2}$, while the density was observed to increase by 25 to $35 \%$ close to the crack (within $10 \mathrm{~mm}$ of the crack), suggesting that the increase in dislocation density is significant as a result of crack growth. However, significantly different trend was observed for Al 1100 (H-14) alloy. In fact, we observe $\approx 7 \%$ decrease in dislocation density close to the crack as compared to the dislocation density away from the crack (see Fig. 5). The density of dislocation $15 \mathrm{~mm}$ away from the crack is approximately $4.5 \times 10^{13} \mathrm{~m}^{-2}$. It should be noted that Eq. 4 gives a relation between dislocation density and $\beta$, assuming dislocations have long range stress. Some dislocations will lower the long range stress by rearrangements, such as by forming dipoles or networks. For Al 1100, we have observed dislocation network formation, presented below, which can under estimate the dislocation density to significant extent.

To study dislocation behavior in the plastic zone, we investigated number of grain boundaries. In $\mathrm{T} 7$ condition, the matrix as well as grain boundary of Al 7075 contains a number of precipitates (see Fig. 6). Some dislocations have been observed to be pinned 
by these precipitates at either side of the grain boundary, Fig. 6(a). In addition, grain boundary can act as sink or source of dislocations during deformation. Fig. 6(b) shows emission of dislocations from a grain boundary. A number of dislocations have also been observed to be pinned (Fig. 6 (c)) and crowded (Fig. 6(d)) at grain boundaries. In Al 1100 , on the other hand, TEM showed dislocation network formation at different grains due to dislocation knitting in the plastic zone (see Figs. 7) as a result of dislocation rearrangement. Fig. 7(a) shows extensive dislocation entanglement of cold rolled Al 1100 before fatigue, and the dislocation configurations change considerably after fatigue (see Fig. 7 (b-e)). We observe three and four fold type of dislocation knitting at different grains in the plastic zone and regions relatively free of dislocations. The dislocations spacing in the network also changes from region to region (see regions 1, 2 and 3 in Fig. 7(c) and regions 1, 2 and 3 in Fig. 7(e)). The minimum spacing observed is approximately $5 \mathrm{~nm}$, corresponding to a maximum tilt of $3^{\circ}$ for a twist boundary. The higher tilt accommodates more dislocation in a network. A higher magnification of a twist boundary containing a network of 3-fold dislocation nodes obtained close to [011] zone is shown in Fig. 8(a). It consists of periodic dislocations. The corresponding fast Fourier transform (FFT) and inverse FFT (IFFT) image are shown in Fig. 8(b and c), respectively. Note that the fatigue crack growth has been carried out at room temperature.

Usually polygonal structure occurs in $\mathrm{Al}$ after deformation at room temperature, and knitting of dislocation takes place on small angle grain boundary when temperature is high enough to allow rapid climb as a result of recovery. In fact, it has been reported that the recovery in $\mathrm{Al}$ takes place at a relatively higher rate. A model on dislocation knitting 
has been put forward by Lindroos and Miekk-oja [26, 27], which requires interaction of forest dislocations with glide dislocations that create junction dislocation by climb. The work required to push dislocations against the repulsive force and to align them is performed by the applied stress. The observation on knitting of dislocation structure suggests dislocation climb occurring at room temperature and the interaction of glide to forest dislocation taking place at different regions/grains in the plastic zone. Note the dislocation configuration on fatigue loading observed in this work in Al 1100 and 7075 is somewhat different from dislocation configurations in low stacking fault energy face centered cubic metals and alloys. For example in $\mathrm{Cu}$ [15] and stainless steel [16], extensive dislocation cell structure consisting of dislocation dipoles instead of dislocation knitting has been observed.

\section{Mechanisms for Lattice Rotations}

Although our aim in this paper is to report and describe in detail the discovery of relatively large rotation near the fatigue crack, we also briefly discuss some possible mechanisms of lattice rotation in fatigue and relate them to our observations. It is well known that in metals and alloys, plastic deformation leads to texture formation, which involves grain rotations. In the monotonic tension test, grains rotate with increasing applied load due to plastic deformation. Relatively high stress in the monotonic tension test activates many slip systems including secondary slip systems on various grains. In deformation by cyclic load, however, deformation usually happens at a much lower applied stress so that only a few primary slip systems are activated. 
More specifically, when a single crystal of a fcc metal is deformed in tension, the slip due to emission of dislocations initially occurs on one of the 111 type planes along 101 type directions, say (111) [101] As the deformation proceeds, the slip direction [101] rotates toward the tensile axis. The grain rotation for a single crystal during monotonic loading is shown schematically in Fig. 9(a). Steps form as dislocations glide in the slip planes to the surface (see case 1 in Fig. 9(a)). Each small step in a slip plane is the multiple of Burgers vector, and the magnitude of total displacement is $n b$, where $\mathrm{n}$ is the number of dislocations and $\mathrm{b}$ is the Burgers vector. However, the segments of crystal rotate toward the loading axis as both ends of the sample during monotonic loading are fixed (see case 2, Fig. 9(a)). One would expect similar trend for polycrystalline specimen during monotonic loading. In fatigue deformation, our results indicate something similar is happening due to emission of dislocations from the crack but localized near the crack.

Thus, in cyclic loading also grains would rotate toward the loading axis (see Fig. 9(b)). One would be able to calculate the magnitude of rotation by knowing the total number of dislocation gliding through the slip planes. Assuming that the total number dislocation produced during crack growth contributes to the total amount of displacement, nb, due to slip. Thus, the average rotation angle, will be given as;

$$
\theta=\tan ^{-1}\left(\mathrm{nb} / 1_{\mathrm{w}}\right)
$$

where $1_{\mathrm{w}}$ is the average width of the grain (see Fig. 9(c)). The average increase in dislocation density in the plastic zone is approximately $30 \%$ (see Fig. 5), suggesting that 
increase in dislocation density is $6.9 \times 10^{12} \mathrm{~m}^{-2}$. The number of dislocations turns out to be $1.6 \times 10^{4}$ for $100 \mu \mathrm{m} \times 25 \mu \mathrm{m}$ pancake size grain. As the grain width, $1_{\mathrm{w}}$, is $25 \mu \mathrm{m}$, the estimated average angle is approximately $11^{\circ}$, which is sufficient to disturb the cube texture close to the crack tip. It should be noted that the dislocation density obtained from $\mathrm{x}$-ray broadening is average over 40 grains. So the estimate of the rotation angle is also an average over those grains. The effect of lattice rotation would be higher closer to the crack. Such a rotation will explain the ratio of the intensity peaks shown in Figs. 2 and 4.

Recently, Shiozawa et al [17] has reported similar observation. They measured the rotation angle spread from individual grains in polycrystalline alloys by diffraction contrast tomography using ultra bright synchrotron radiation both in the monotonic tension test and in fatigue tests. In fatigue tests, they found that the rotational spread changed in particular grains and particular diffraction planes only. What is more interesting is the finding that the diffraction plane in which the greatest change in the rotation angle spread was observed coincided with the slip plane for the material which was commercially pure iron. This is consistent with the experimental observations that the relative intensity of (111) increases as compared to (200) as we approach the crack, suggesting that slip in the 111 plane in $\mathrm{Al}$ is associated with lattice rotation. It is also known that the increase in spread of the rotation angle is related to the increase in dislocation density possibly due to slip near the crack [17]. The stress due to crack tip is expected to be low in fatigue, so only primary slip system is activated in individual grain. As mentioned before any slip of this nature will increase the rotation angle also. In Al 
systems, the recovery and rearrangements of dislocations will reduce the spread of rotation angle to some extent unlike the case of $\mathrm{Fe}$ where the rearrangement of dislocations at room temperature is insignificant.

Some additional mechanisms of grain rotation are now considered. The recovery process in $\mathrm{Al}$ and its alloys can lead to subgrain formation. These subgrains can also in principle rotate under load, which can then add to grain rotation angle value. Cheng et al, [11] using high resolution TEM showed that in a nanocrystalline Ni-Fe alloy under cyclic deformation, the grain coarsening may have been generated through the lattice reorientation under the high stress concentration near crack tip, and this process is assisted with the dislocation accumulation during the fatigue process. They also showed that the dislocations lodged in grain boundaries cause rotations across the grain boundaries and, in the larger grains, and also in some cases resulting in a secondary subgrain structure. It is not clear whether this mechanism will be operating in relatively large size grains in our case.

In addition, the recrystallization texture of cold rolled high purity Al sheet is predominantly cube orientation $(\{100\}<001>)$. In commercial pure $\mathrm{Al}$, in addition to cube orientation, R-fiber $(\{123\}<634>)$ has been observed [25,26]. For Al alloys, in addition to cube orientation, other components, such as gross $(\{101\}<010>)$, brass $(\{101\}<121>)$, copper $(\{112\}<111>)$ and $\mathrm{P}(\{011\}<122>)$ have also been reported upon annealing $[28,29]$. However, we observe a gradual increase in $\{111\}$ orientation close to the crack for $\mathrm{Al} 7075$ (T7) and $\mathrm{Al} 1100$ (H14), suggesting that the observed lattice 
rotation is not due to recrystallization or recovery during the fatigue process at room temperature. Note the recrystallization temperature of Al 7075 is significantly higher than room temperature.

Another contributory factor to the grain rotation could be due to a change in the content of grain boundary (GB) dislocations [9]. Such a change of GB dislocations must change GB angle between neighboring grains, as given by well-known Frank-Bilby equation. The change in dislocation content is due to the accumulations of dislocations emitted during cyclic loading. We believe that the rotation depends on the manner in which dislocations are distributed in a grain. In low-stacking fault energy materials, such as stainless steel and $\mathrm{Cu}$, as the redistribution of dislocations leads to cell structures $[15,16]$ made of dislocation dipoles in the plastic zone, it may not significantly contribute to lattice rotation because dislocations are immobile. However, in high-stacking fault energy material, such as $\mathrm{Al}$, the rearrangement of dislocation is not in form of dislocation cell structure, particularly in al 7075 , and it leads to significant lattice rotation in the plastic zone as mentioned.

\section{Summary and Conclusions}

We report for the first time a significant lattice rotation in the plastic zone over several grains ahead of a fatigue crack tip at room temperature in Al 1100 (H14) and Al-7075 (T7). This lattice rotation was observed by employing X-ray diffraction (XRD). A series of high resolution XRD scans measured at different locations in the vicinity of crack tip 
showed variations in the relative intensities of 111, 200 and $220 \mathrm{Al}$ peaks. The intensity of 111 peaks was observed to gradually increase as compared to that of the 200 peaks as we approach the crack, suggesting lattice rotation as a result of fatigue crack growth at room temperature. The texture changes gradually from cubic away from the crack to more random orientation close to the crack, which has been quantitatively assessed using March-Dollase approach. Transmission electron microscopy (TEM) was performed in the same regions to confirm the results and to also observe the changes in microstructure. We observe a significant change in dislocation density and a change in dislocation configuration in the plastic zone. We ascribe the lattice rotation to the increase in dislocation density in the plastic zone. The estimated average angle of rotation is approximately $11^{\circ}$, which is sufficient to disturb the cube texture close to the crack. After considering many possibilities we conclude that bulk of lattice rotation must be ascribed to glide of large number of dislocations along $\{111\}$ planes. It should be noted that such lattice rotations will constantly alter fatigue parameters during crack growth, and may even change fatigue mode. These changes are expected to seriously impact fatigue life prediction of materials. In addition, the observed plastic zone size for Al 7075 obtained from the lattice rotation is an order of magnitude higher than the calculated plastic zone.

\section{Acknowledgment}

Funding for this project was provided by the Office of Naval Research (ONR) through the Naval Research Laboratory's 6.1 Research Program.

\section{References}


1. R. C. Gifkins, Grain-boundary sliding and its accommodation during creep and superplasticity, Metall. Trans. A, 7 (1976) 1225-1232.

2. M. F. Ashby, G. H. Edward, J. Davenport and R. A. Verrall, Application of bound theorems for creeping solids and their application to large strain diffusional flow, Acta Metall., 26 (1978) 1379-1388

3. M. G. Zelin and A. K. Mukherjee, Geometrical aspects of superplastic flow, Mater. Sci. Eng. A, 208 (1996) 210-225

4. F. R. N. Nabarro, Steady state diffusional creep, Phil. Mag. A., 16 (1967) 231237

5. D. Lee, The strain rate dependent plastic flow behavior of zirconium and its alloys, Metall. Trans., 1 (1970) 1607-1617

6. J.C.M. Li, Possibility of subgrain rotation during recrystallization, J. Appl. Phys., 33 (1962) 2958-2965

7. J. Schiotz, F. D. Di Tolla and K. W. Jacobsen, Softening of nanocrystalline metals at very small grain sizes, Nature, 391 (1998) 561-563.

8. J. Schiotz and K. W. Jacobson, A maximum in the strength of nanocrystalline copper, Science, 301(2003) 1357-1359

9. L. Wang, J. Teng, P. Liu, A. Hirate, E. Ma, Z. Zhang, M. Chen, X. Han, Grain rotation mediated by grain boundary dislocations in nancrystalline platinum, Nature communication, 5 (2014) Art. No. 4402

10. L. Margulies, G. Winther, H. F. Poulsen, In Situ Measurement of Grain Rotation During Deformation of Polycrystals, Science, 291 (2001) 2392-2394 
11. S. Cheng, Y. Zhao, Y. Wang, Y. Li, X. Wang, P. K. Liaw, and E. J. Lavernia, Structure Modulation Driven by Cyclic Deformation in Nanocrystalline NiFe, Phys. Rev. Lett.,104, (2010) 255501

12. I. A. Ovid'ko and A. G. Sheinerman, Special rotational deformation in nanocrystalline metals and ceramics. Scripta Mater. 59 (2008) 119-122.

13. D. Farkas, S. Mohanty, J. Monk, Linear grain growth kinetics and rotation in nanocrystalline Ni. Phys. Rev. Lett. 98 (2008) 165502

14. V. Yamakov, D. Wolf, S. R. Phillpot, A. K. Mukherjee and H. Gleiter, Deformation-mechanism map for nanocrystalline metals by molecular dynamics simulation, Nat. Mater., 3 (2004) 43-47.

15. K. Katagiri, A. Omura, K. Koyanagi, J. Awatani, T. Shiraishi, H. Kaneshiro, Early stage in fatigued crack tip dislocation morphology in fatigued copper. Met. Trans. A, 8 (1977) 1769-1773

16. M. S. Pham, C. Solenthaler, K. G. F Janssens, S. R. Holdsworth, Dislocation structure evolution and its effects on cyclic deformation response of AISI 316L stainless steel. Mat. Sci. Engg. A, 528 (2011) 3261-3269

17. D. Shiozawa, Y. Nakai, R. Miura, N. Masada, S. Matsuda and R. Nakao, 4D evaluation of grain shape and fatigue damage of individual grains in polycrystalline alloys by diffraction contrast tomography using ultrabright synchrotron radiation, International J. of Fatigue, 82 (2016) 247-255.

18. L.N. Brewer, M.A. Othon, L.M. Young and T.M. Angeliu, Misorientation Mapping for Visualization of Plastic Deformation via Electron Back-Scattered Diffraction, Microscopy and Microanalysis, 12 (2006) 85-91 
19. A. Irastorza-Landa, H. Van Swygenhoven, S. Van Petegem, N. Grilli, A. Bollhalder, S. Brandstetter, D. Grolimund, Following dislocation patterning during fatigue, Acta Mater., 112 (2016) 184-193

20. E. Zolotoyabko, Determination of the degree of preferred orientation within the March-Dollase approach, J. Appl. Cryst., 42 (2009) 513-518

21. S. S. Harsha, Joseph. S. Melinger, S. B. Qadri and D. Grischkowsky, Substrate dependence of $\mathrm{THz}$ vibrational modes of polycrystalline thin films of molecular solids in waveguide THz-TDS, J. Appl. Phys. 111 (2012) 023105

22. S. T. Rolfe and J. M. Barsom, Fracture and Fatigue Control in Structures, Application of Fracture Mechanics, (1977) Prentice Hall, Inc. Englewood Cliffs, New Jersey, p. 42.

23. P. Gay, P. B. Hirsch and A. Kelly, The estimation of dislocation densities in metals from X-ray data, Acta Metall., 1 (1953) 315-319

24. M. J. Hordon and B. L. Averbach, X-ray measurements of dislocation density in deformed copper and aluminum single crystals, Acta Metal., 9 (1961) 237-246 (1961).

25. S. B. Qadri and J. H. Dinan, X-ray determination of dislocation density in epitaxial ZnCdTe, Appl. Phys. Lett., 47 (1985) 1066-1068

26. H. M. Miekk-Oja and V.K. Lindroos, The formation of dislocation networks, Surface Science, 31 (1972) 422-455 
27. V. K. Lindroos and H. M. Miekk-Oja, Knitting of dislocation networks by means of stress-induced climb in aluminium-magnesium alloy, Phil. Mag., 17 (1968) $119-133$

28. X. Wen, W. Wen, Y. Zhang, B. Xu, Q. Zeng, Y. Liu, L. Tong, T. Zhai and Z. Li, The Effect of Fe Content on Recrystallization Texture Evolution, Microstructures, and Earing of Cold Rolled Continuous Cast AA5052 Alloy Sheets, Metall. and Mater. Trans. A, 47 (2016) 1865-1880

29. S. Gourdet, F. Montheillet, An experimental study of the recrystallization mechanism during hot deformation of aluminum, Mat. Sci. Engg. A, 283 (2000) $274-288$ 


\section{Figure Captions}

Fig. 1. (a) The fatigue growth rate as a function of $\Delta K$. (b) The part of the CT specimen showing the crack originated at the notch.

Fig. 2. A series of XRD diffraction patterns from Al 7075-T731 at different locations. Here d denotes the distance from the crack.

Fig. 3. XRD map using the relative 111 peak intensity of Al 1100 around the crack.

Fig. 4. Integrated intensity ratio of 111 to 200 as a function of position from crack at either side of the crack for Al 7075 (right scale) and Al 1100 (left scale).

Fig. 5. The dislocation density as a function of distance from crack obtained the peak width at FWHM of 111 peak as a function of distance from crack for Al 7075 and $\mathrm{Al}$ 1100.

Fig. 6 (a-d) Dislocation configurations at different grains in the plastic zone for Al 7075.

Fig. 7. Dislocation configurations for Al 1100 before fatigue (a) and after fatigue in the plastic zone at different grains (b-e), showing dislocation network formation.

Fig. 8. (a) A higher magnification TEM image of a twist boundary containing a network of 3-fold dislocation nodes. (b) The fast Fourier transform (FFT) from the square box. (c) An IFFT) image showing the dislocations.

Fig. 9. (a) Schematic diagrams showing the lattice rotation during monotonic loading, case-1 and case-2. (b-c) Schematic diagrams showing the lattice rotation across several grains in fatigue loading and the rotation angle, $\theta$, respectively. 


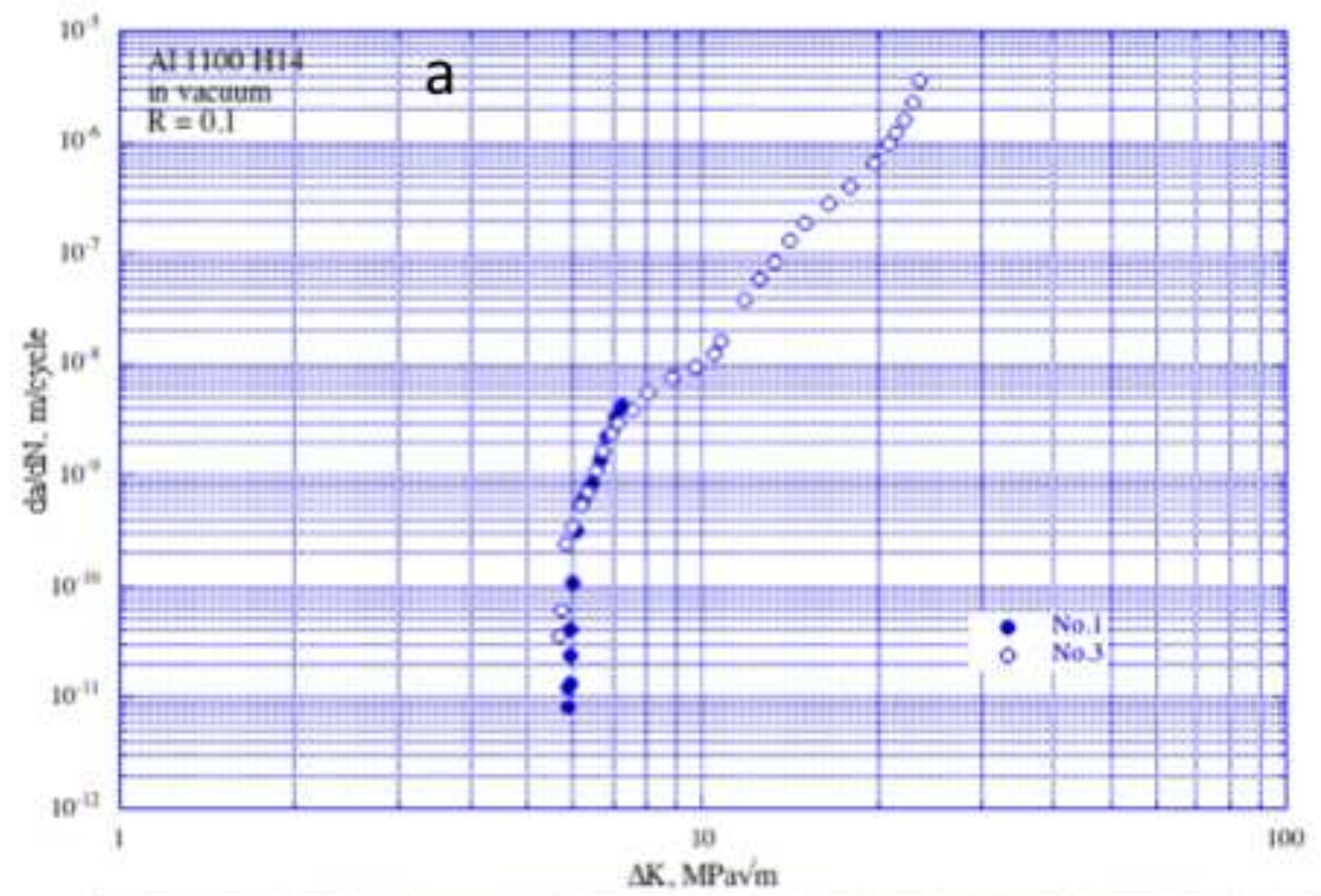

Fig. 1

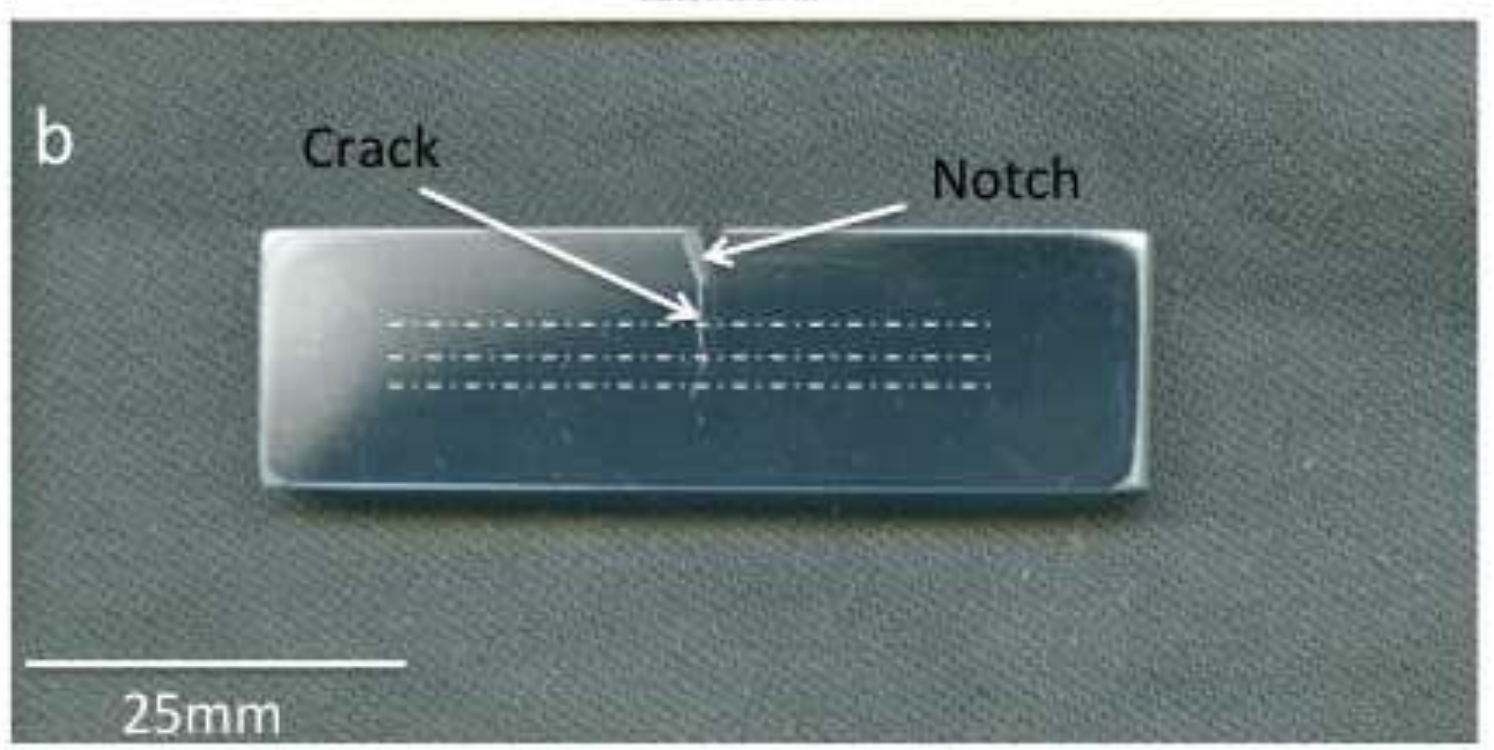




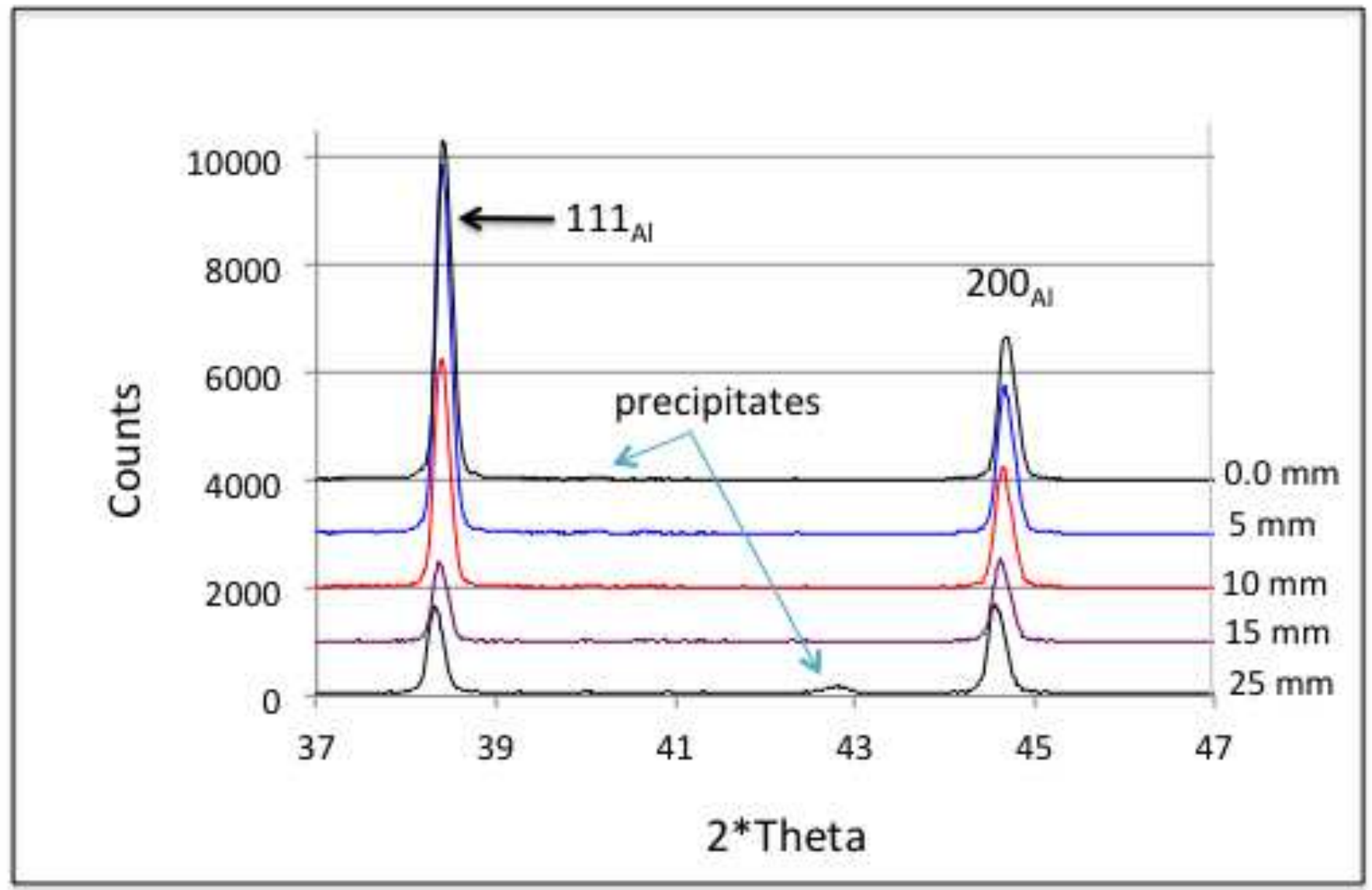

Fig. 2 


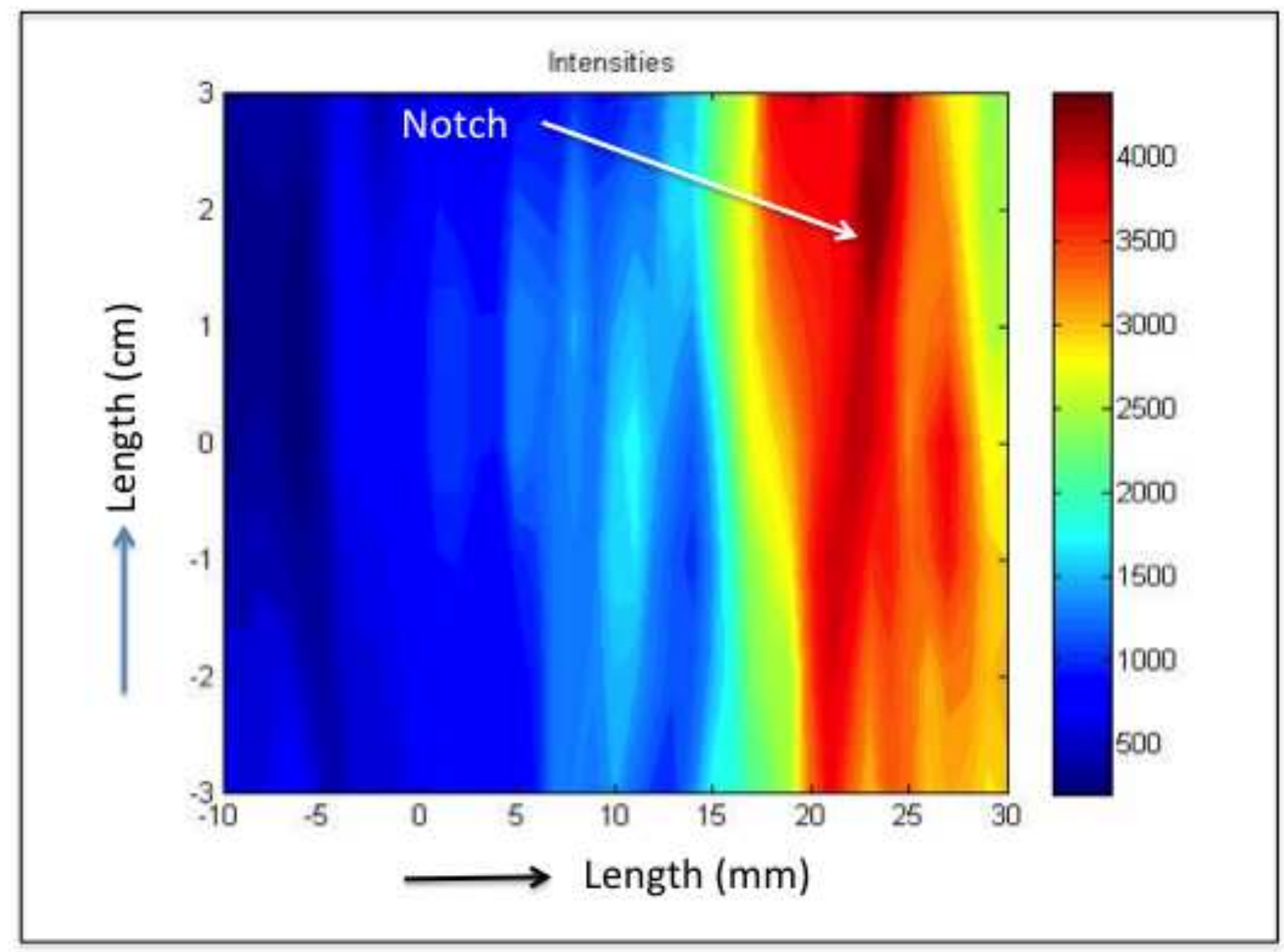

Fig. 3 


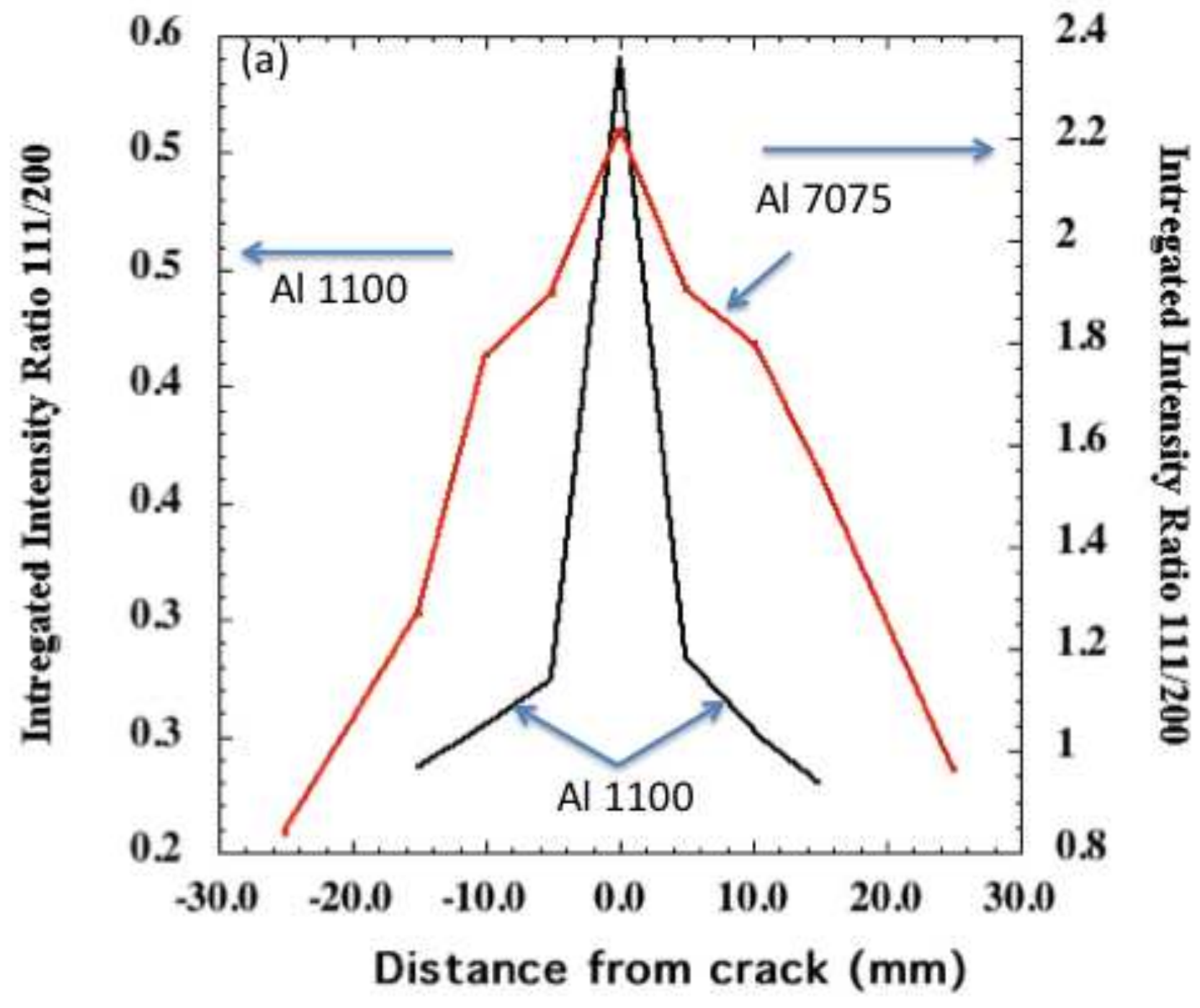

Fig.4 


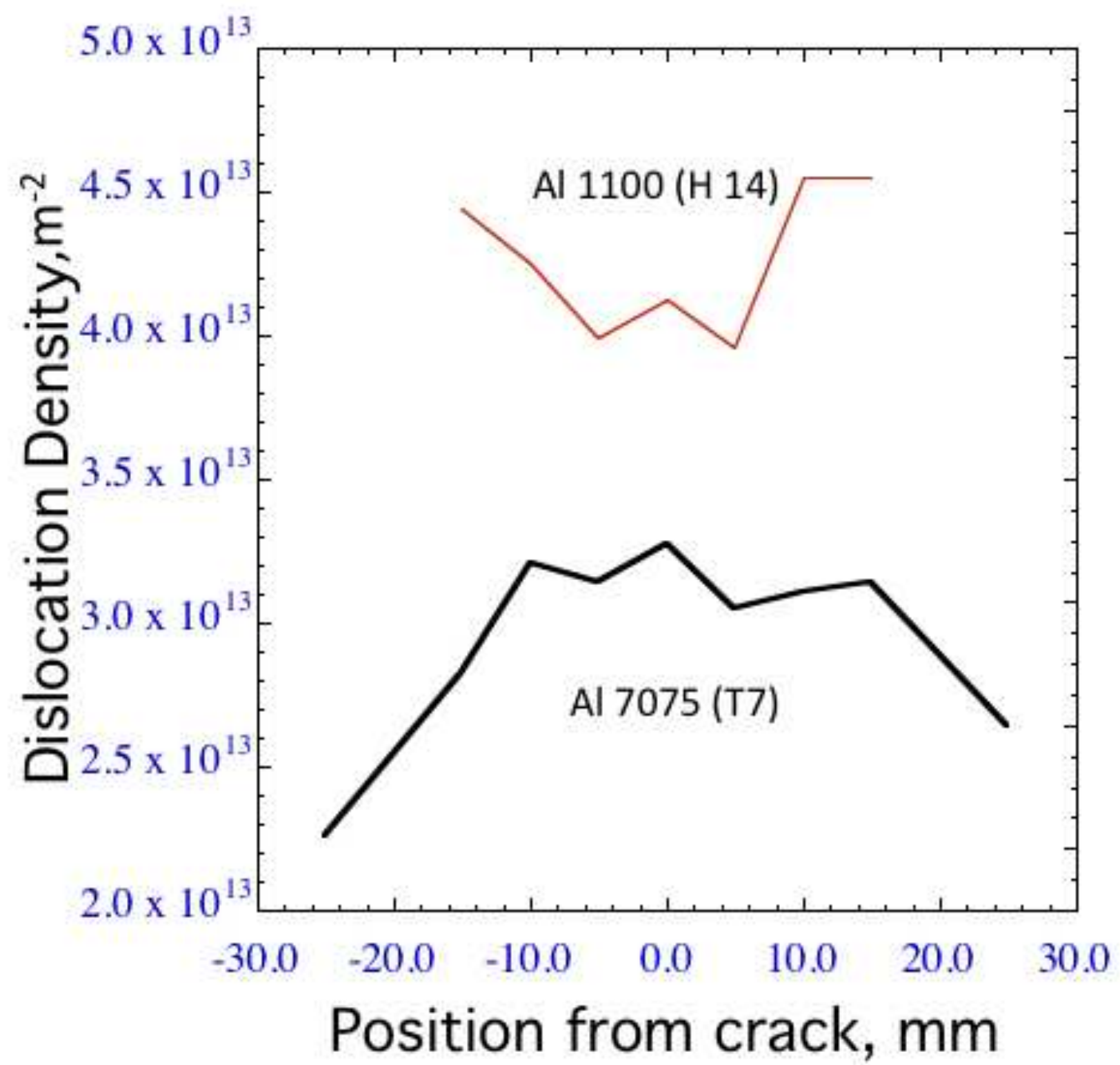

Fig.5 

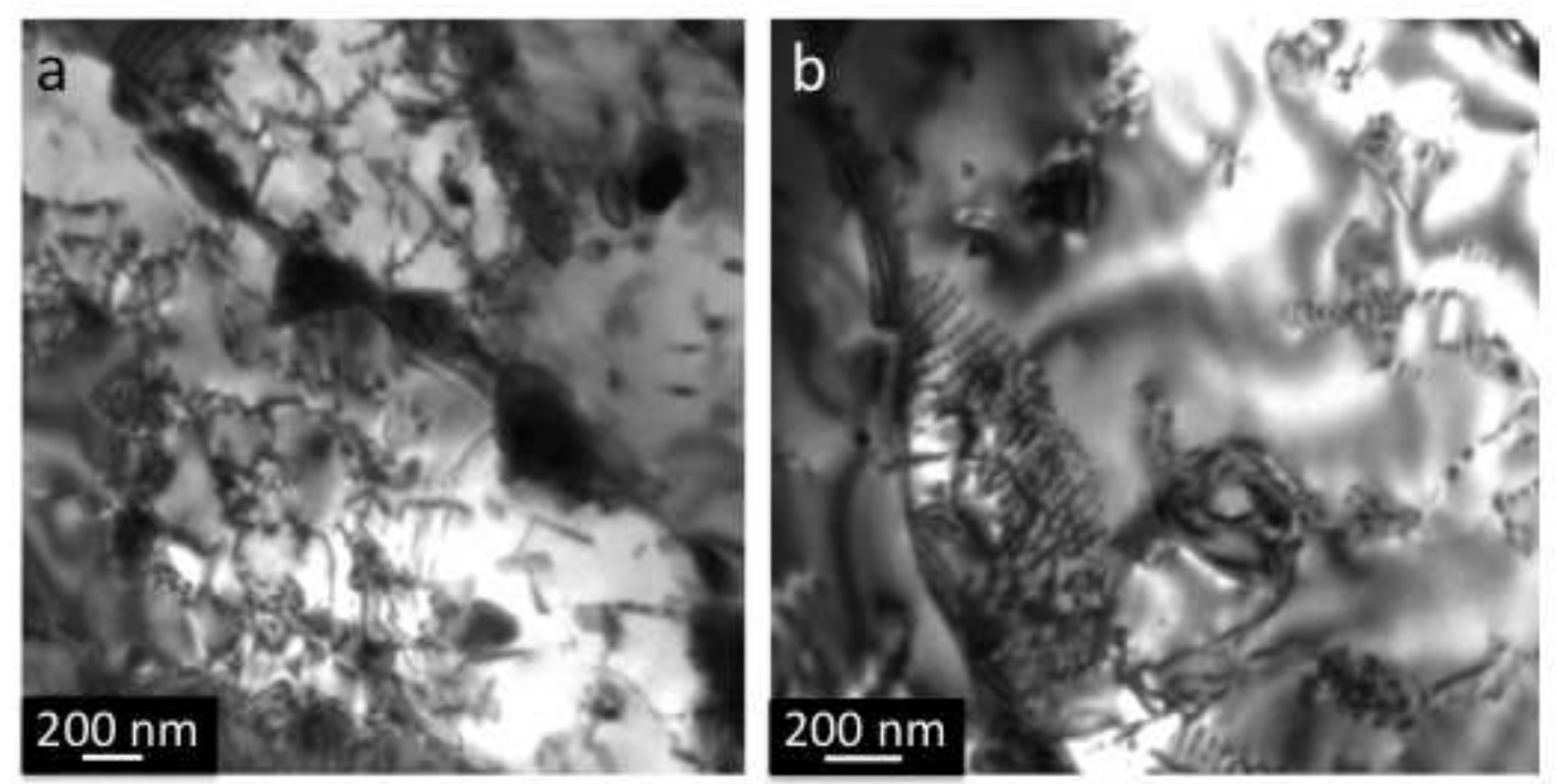

Fig. 6
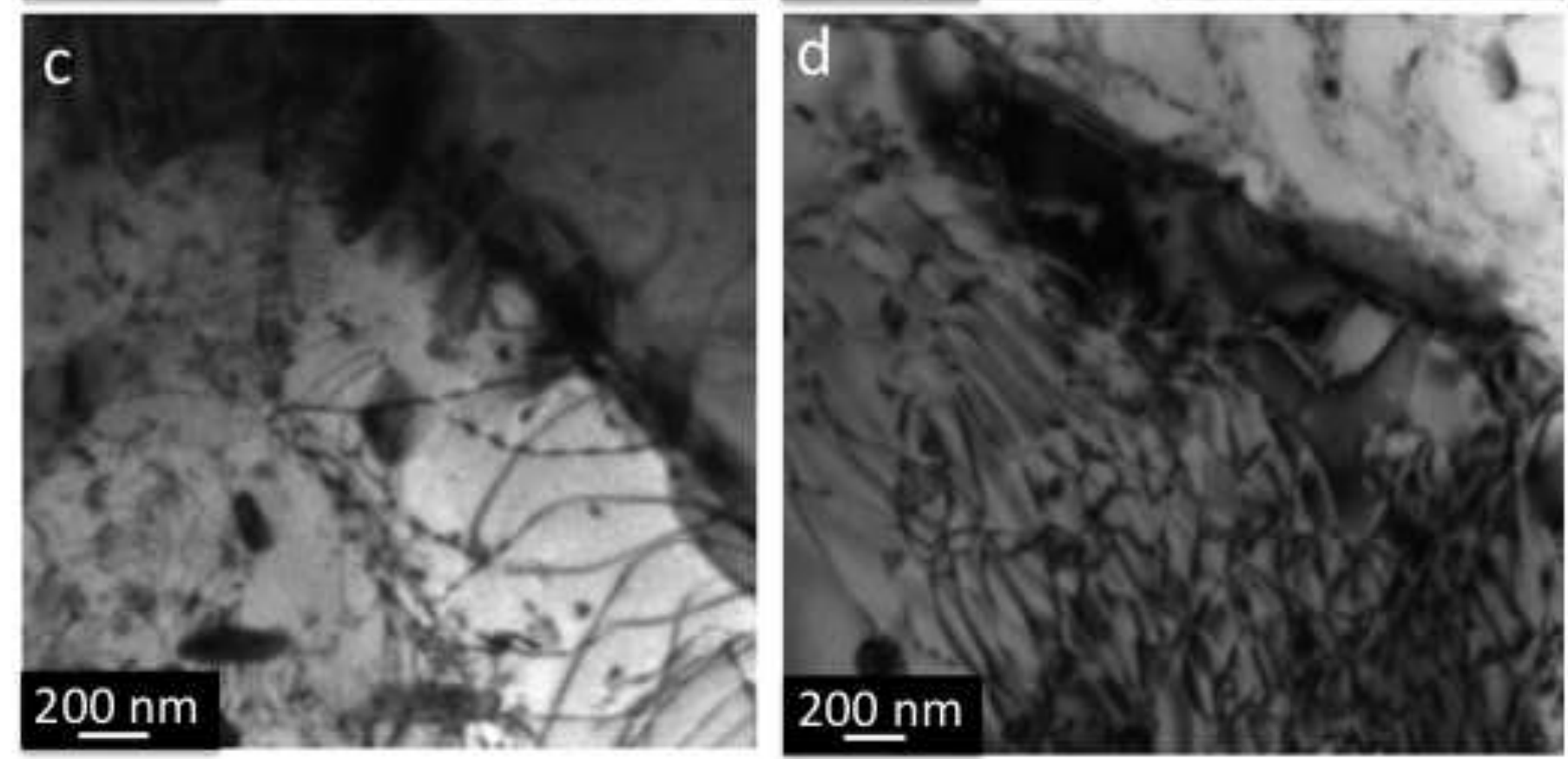

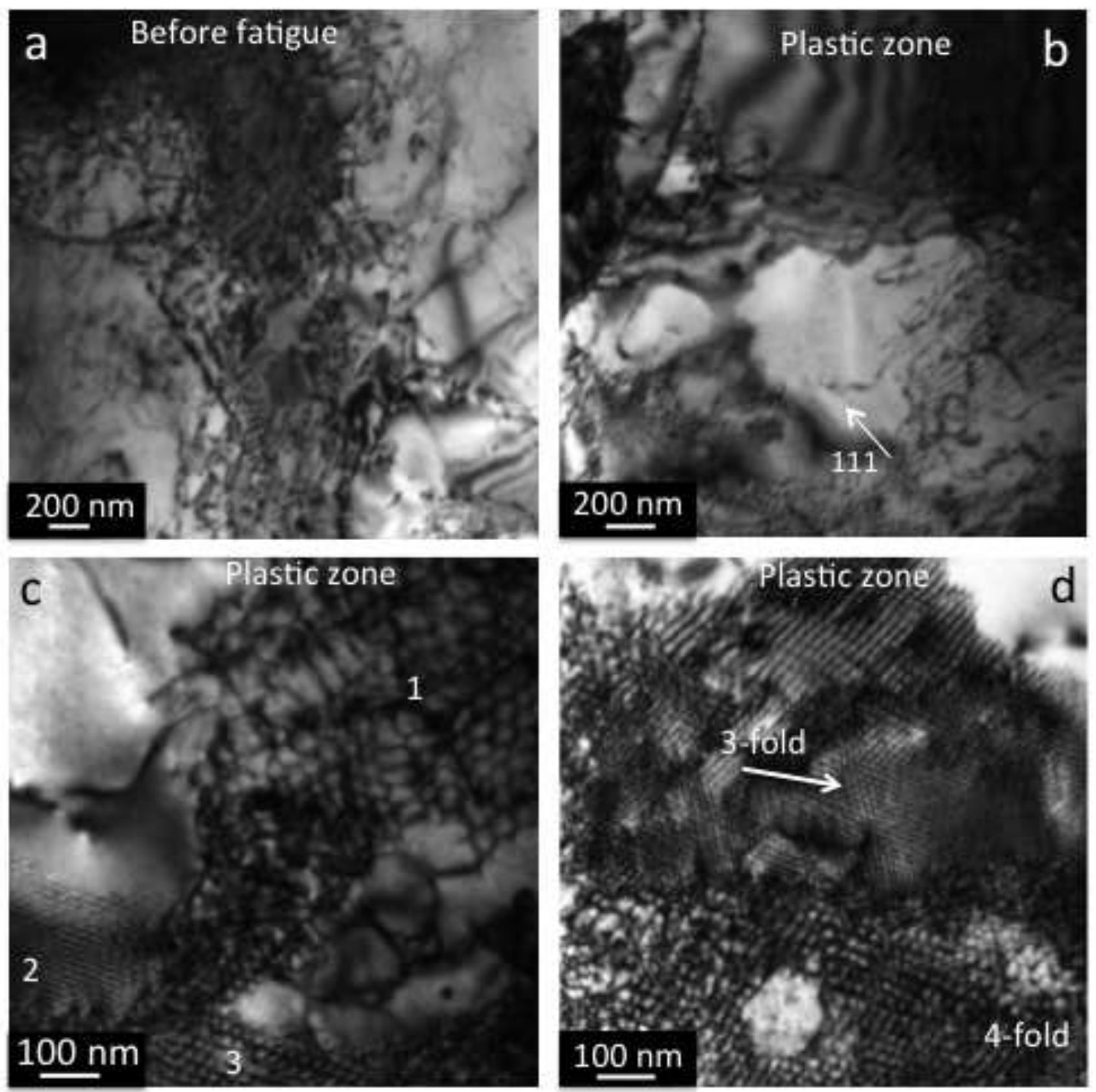

Fig.7 


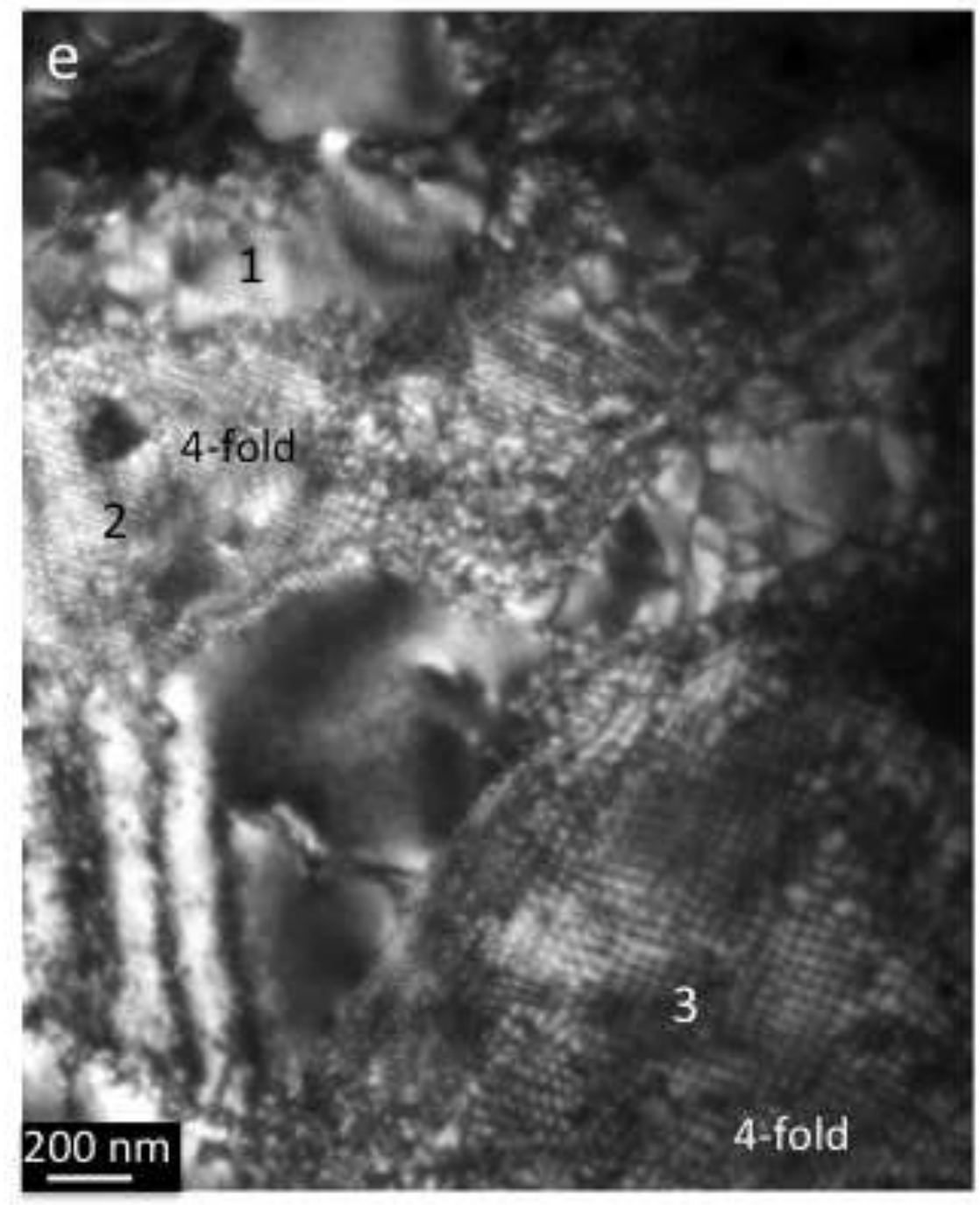

Fig.7 

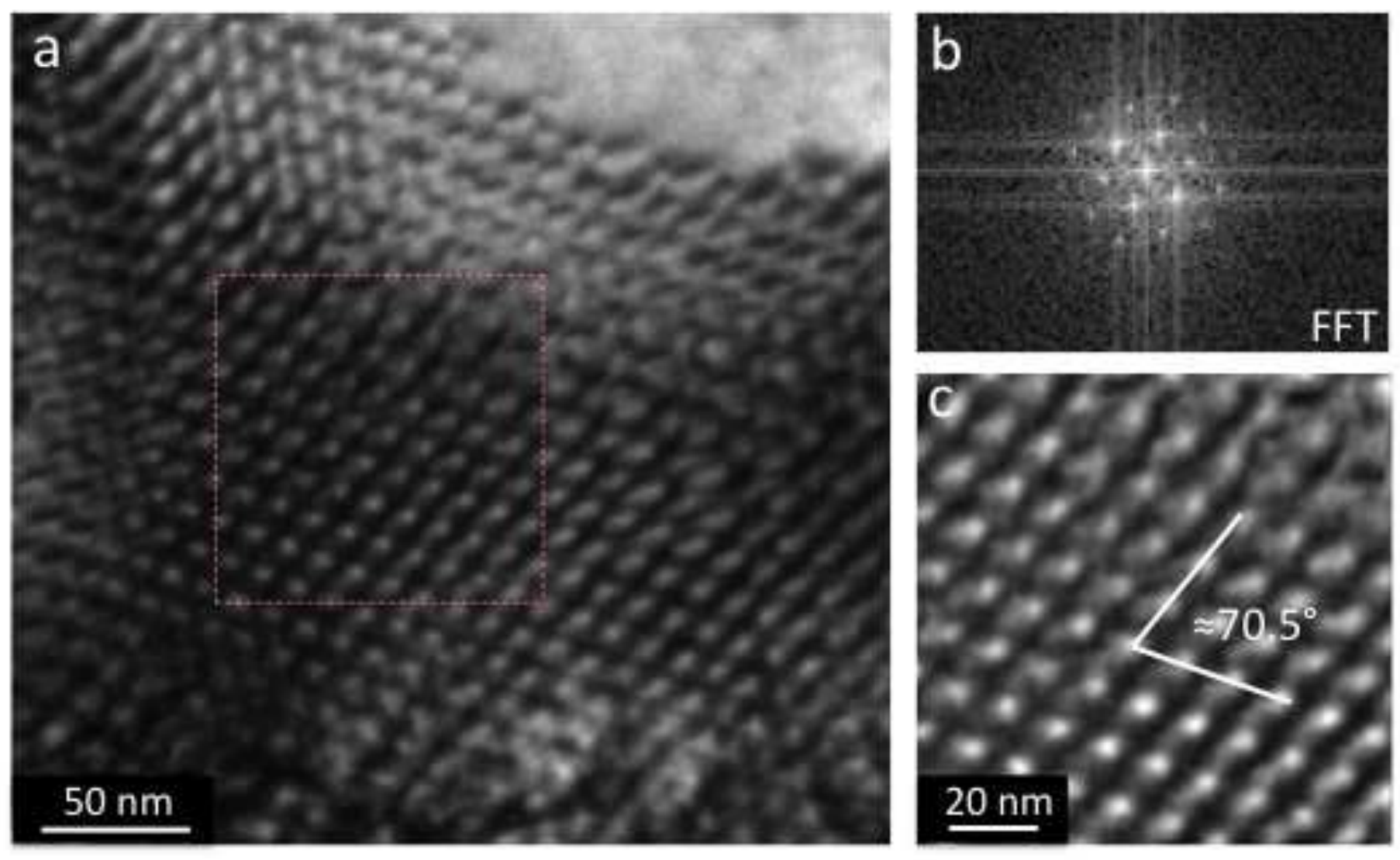

Fig. 8 

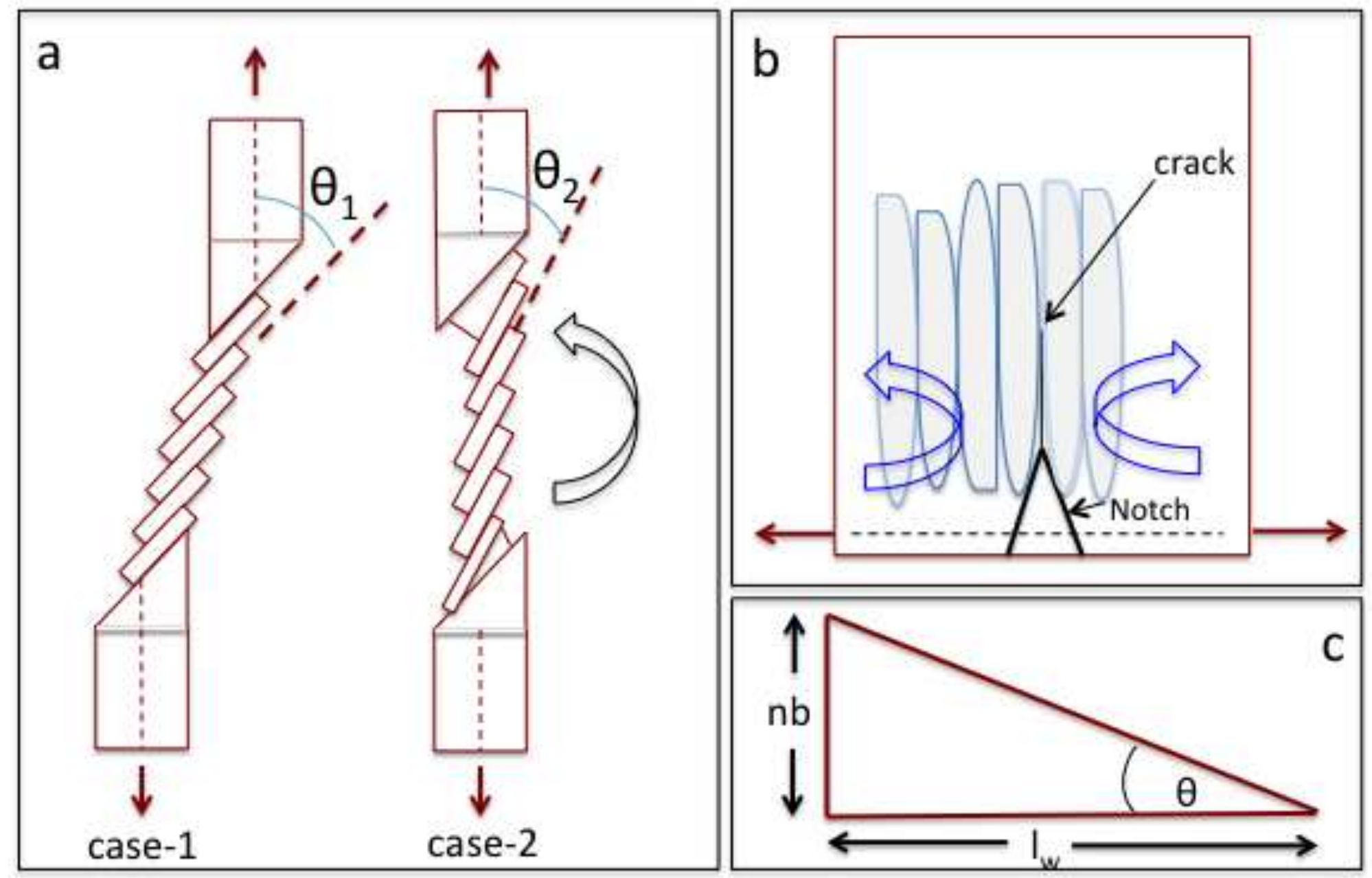

Fig.9 


\title{
Fatigue Mediated Lattice Rotation in Al Alloys
}

\author{
R. Goswami ${ }^{1}$, S. B. Qadri and C.S. Pande
}

Division of Materials Science and Technology, Naval Research Laboratory, Washington DC 20375

\begin{abstract}
A significant lattice rotation in the plastic zone of a fatigue crack at room temperature in highly oriented Al 1100 and Al 7075 alloys has been observed by employing X-ray diffraction (XRD) and transmission electron microscopy (TEM). The intensity of 111 peak gradually increases as compared to the intensity of 200 peak as we approach the crack, suggesting lattice rotation. The texture was seen to change gradually from the cubic, away from the crack, to a more random orientation close to the crack. We ascribe the lattice rotation to the increase in dislocation density in the plastic zone. The estimated average angle of rotation is approximately $11^{\circ}$, which is sufficient to disturb the cube texture close to the crack. In addition, the observed plastic zone, as estimated by the lattice rotation, is order of magnitude higher as compared to the theoretically predicted plastic zone. This is of great importance, as it leads to a new insight of the underlying physics of the deformation process in the plastic zone under cyclic loading.
\end{abstract}

Key words: Lattice Rotation, Microstructure, Dislocations networks, Twist boundaries, TEM, Fatigue Crack growth

\footnotetext{
${ }^{1}$ Corresponding Author: Ramasis Goswami, e-mail: ramasis.goswami@nrl.navy.mil
} 